\title{
Tips and Tricks to Manage Vascular Risks Using the Transnasal Endoscopic Approach to Pituitary Adenomas
}

\author{
Chinezu Rareș ${ }^{1,2^{*}}$, Berhouma Moncef ${ }^{3}$, Jacquesson Timothée ${ }^{3}$, Raverot Gerard ${ }^{4,5,6}$, Jouanneau \\ Emmanuel $3,5,6$ \\ University of Medicine and Pharmacy Tirgu Mures, Tirgu Mures, Romania \\ 2 Clinic of Neurosurgery, County Clinical Emergency Hospital Tirgu Mures, Romania \\ ${ }^{3}$ Service de neurochirurgie B, Unité de chirurgie de la base du crâne. Groupement hospitalier Est, Hospices civils de Lyon, Bron, France \\ 4 Fédération d'endocrinologie du pôle Est, Aile A1, Groupement hospitalier Est, Hospices civils de Lyon, France \\ 5 Université Claude-Bernard, Lyon-1, Lyon, France \\ 6 Inserm U1028, CNRS UMR 5292, Centre de neurosciences de Lyon, France
}

Objective: The purely transnasal endoscopic approach has taken in the last decade a paramount importance in the treatment of pituitary adenomas, but some authors have been linked it to an increase in vascular complications. The aim of this paper is to provide a stepwise description of the vascular risks and steps required to avoid them. Method: We present the minimal invasive technique used by the senior author in over 900 transnasal purely endoscopic approaches focusing on the relevant vascular landmarks, preoperative and operative steps taken in order to avoid vascular injury and the management of vascular injury from a multitude of sources. Conclusion: Endoscopy has brought a wider field of view, with numerous vascular structures well inside the operating field. This represents control but also higher risk. Solid knowledge of the skull base anatomy and proper preoperative imaging coupled with an experienced surgeon can greatly reduce the vascular risks associated with surgery.

Keywords: pituitary adenomas, endoscopy, vascular, skull base

Received: 18 August 2015 / Accepted: 03 September 2015

\section{Introduction}

The introduction of the purely endoscopic transnasal technique in the treatment of pituitary adenomas 20 years ago by Jho et al. [1] was a important moment for the surgery of pituitary adenomas. In the following decades the technique has seen wide acceptance and has taken a paramount importance in the treatment of pituitary adenomas [2-5].

Recently some authors have compared with the use of meta-analysis the purely endoscopic with previously published microscopic series and have reported an increase of vascular injury in the endoscopic series [6].

The statistical techniques used to generate these metaanalytical results have been subsequently dismissed by other authors $[7,8]$ but it is beyond doubt that knowledge of vascular risks and their management should be in the armament of every surgeon performing endoscopic pituitary surgery.

The aim of this paper is to present the purely endoscopic transnasal technique used by the senior author (EJ) at the Endoscopic and Skull Base Unit of the Pierre Wertheimer Hospital, Lyon, France, in more than 900 cases of pituitary adenomas, emphasizing on how to prevent and manage the vascular complications or events.

* Correspondence to: Rareș Chinezu

E-mail: rchinezu@yahoo.com

\section{Relevant anatomy focusing on vascular risks and landmarks}

Endoscopic approaches to the pituitary require an in depth knowledge of the nasal anatomy, facial aeric sinuses and skull base. It is recommended that, at least during the initial phase of the learning curve of these procedures, the neurosurgeon should be accompanied by an ENT surgeon $[9,10]$. Many anatomical studies have been previously published [11-17] as such we will succinctly present the important landmarks and vascular risks of each surgical phase.

\section{Nasal compartiment}

The choana represents the most important anatomical landmark of the nasal cavity and its identification should be performed as a first step in all endoscopic transnasal approaches. Superior to the choana at about $1,5 \mathrm{~cm}$ the sphenoid ostium can be found (Figure 1).

The delicate and well-vascularized nasal mucosa and the nasoseptal branches of the sphenopalatine artery, which pass immediately below the sphenoidal ostium, represent the vascular risk in the nasal compartment.

\section{Sphenoid sinus}

All endoscopic approaches to the sella turcica get throught the sphenoid sinus. The anatomy of the sphenoidal sinus is highly variable and can be divided by one or more vertical 
septations that are often asymmetric. In some cases these septations can be used in identifying the positions of the carotids [14].

According to the pneumatisation of the sphenoid sinus three types have been described: the sellar type (75\%) with pneumatisation reaching the clivus and facilitating transsphenoidal surgery, the presellar type $(20 \%)$ with a moderate pneumatisation in which the posterior border of the sinus does not go beyond the lower half of the sellar floor, and the conchal type (5\%), in which a very small sinus is separated by the sella by a thick bony wall, requiring extensive drilling [18].

After removing the septa, the following landmarks are identified (Figure 2):

- up and anteriorly the tuberculum of the sella and the planum sphenoidale

- centrally, the sella and the pituitary fossa.

- laterally and up the optic nerve, paraclinoid carotid processes (C5) and the medial and lateral optico-carotid recesses.

- laterally, the anterior wall of the cavernous sinus and below the paraclival carotid processes (C3).

- below the clivus corresponding posteriorly to the brainstem.

Anatomic and vascular checkpoints related to the internal carotid artery

The paraclival, parasellar and paraclinoid segments of the internal carotid artery (ICA) are laying facing the lateral wall of the sphenoid sinus. The characteristic curvature of the C3 - C5 segments produces a bulging on the surface of the sphenoid sinus, and depending on the pneumatisation of the sphenoid sinus this can vary from a small bulge to a complete outline of the vascular course. In most of the

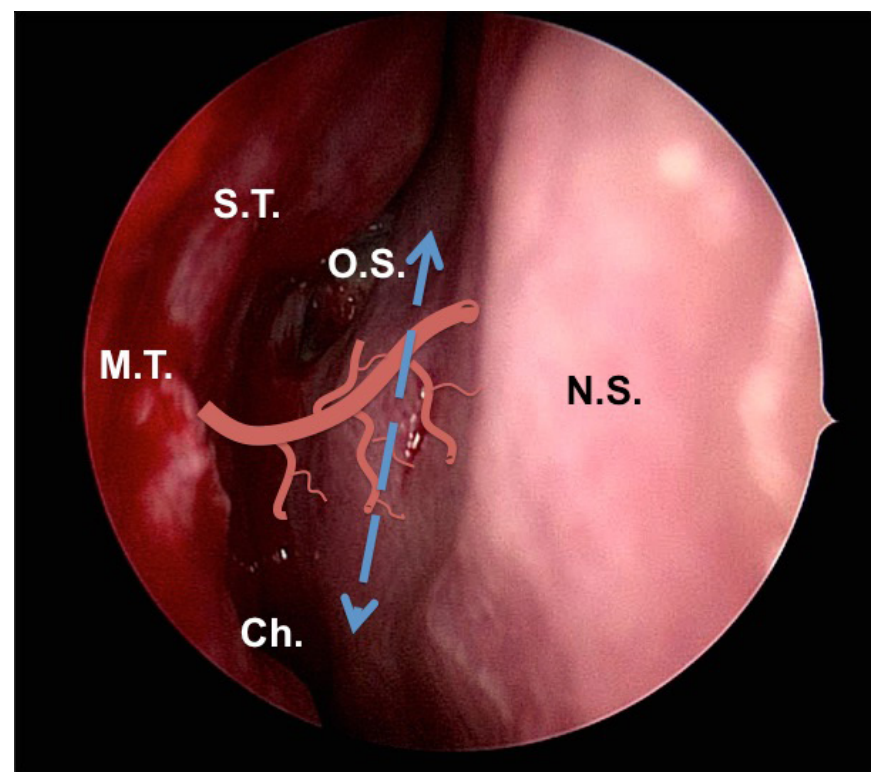

Fig. 1. Vascular projection of the nasoseptal branch of the sphenopalatine artery in the right nostril. M.T. lateralized middle turbinate, Ch - choanal arch, S.T. - superior turbinate, N.S. - nasal septum, dashed line - area of coagulation before mucosal dissection. cases bulging of the $\mathrm{C} 3$ and $\mathrm{C} 5$ segments will be visible. These bony prominences are the most important landmarks of the sphenoidal sinus. Care should be taken as there are anatomical variations in which the bone covering the carotid canal can miss partially or completely especially on the C5 segment [19].

Of extremely great importance for the operating surgeon is to localize the paraclinoidian carotid segment (C5), as this is the point where the intercarotid distance is the shortest and were the bone may be thinnest. (Figure 2).

\section{Sellar and intrasellar anatomy}

Anatomic sellar and vascular variants related to tumor size and type

The size and functional type of the adenoma can produce a large variety of modifications in the anatomy of the sella.

In the case of large adenomas or in elderly patients the thickness of the bone covering the sella, the cavernous sinus, C5 segment and the optic nerve can be extremely thin; sometimes bone can be reduced to the constancy of an eggshell.

In the case of patients suffering from acromegaly the existence of a constant reduction of the $\mathrm{C} 5$ inter-carotid space has been proven [20]. In some cases the inter-carotid distance can be reduced to just $0.5 \mathrm{~mm}$, the so-called "kissing carotids". (Figure 3.)

Venous structures are normally very rich in and around the sella. This may cause bleeding issues especially in microadenomas mainly corticotroph ones. In such situations, the size of the sella is small, leaving the surgeon with a limited working field. Due to a lack of compression the anterior and posterior intercavernous venous plexus are patent. In the opposite situation of macroadenomas, tumor compression leads to disappearance of venous vessels.

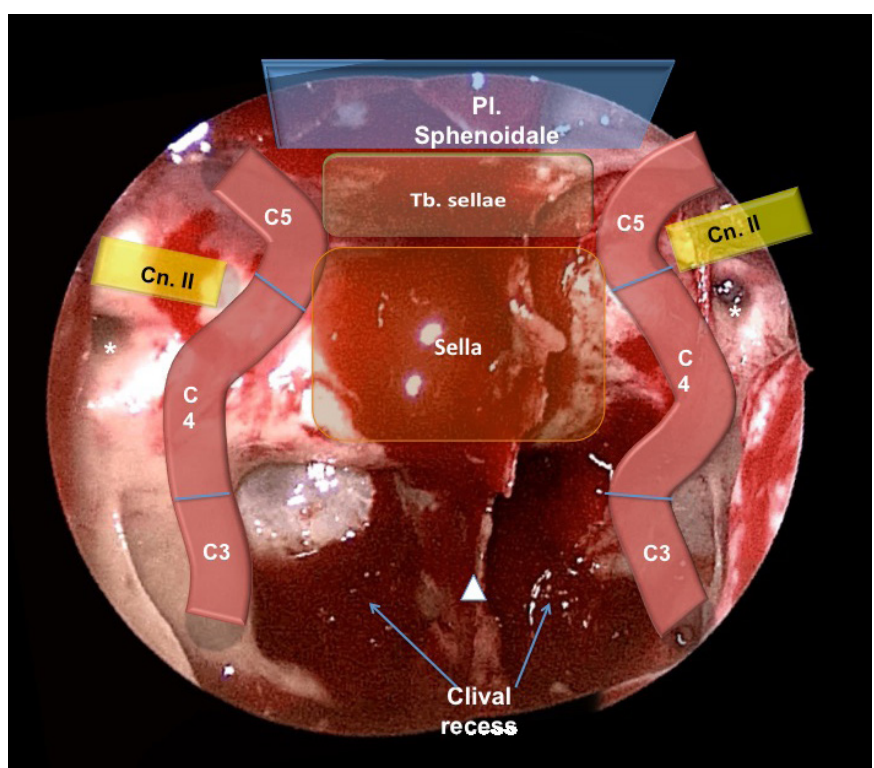

Fig. 2. Panoramic view of the sphenoidal skull base landmarks. (Stitched multiple images)

* - lateral optico-carotidian recess, arrowhead - vertical septum of the sphenoidal sinus (panoramic image generated with ICE software, Microsoft Research, USA) 
Intrasellar carotid artery procidence, vascular malformation and vestigial remnants

The distance between the lateral margin of the pituitary and the medial part of the ICA varies form 1 to $7 \mathrm{~mm}$, but there are cases described were the carotid exits the cavernous sinus and protrudes in the pituitary gland [11] (Figure 3).

The persistent trigeminal artery is the most common remnant of the embryonic carotid-basilar trunk. In 50\% of cases it penetrates the sella, runs along an own vascular groove and then perforates the dura near the clivus in order to join with the basilar artery. The coexistence of the persistent trigeminal artery and a pituitary adenoma is extremely rare, and to date only 7 cases have been reported in the literature [21].

Pituitary failure due to an intra sellar carotid aneurysm is rare [22], but the presence of an ovoid intrasellar mass in continuation with the cavernous sinus should raise suspicions.

The coexistence of a pituitary adenoma with an intrasellar aneurysm extremely rare, but seems to appear more often with GH secreting adenomas [23].

The ICA route and abnormal vascular signal can be perfectly studied on pre operative MRI and angiography should be performed in all cases with suspicion.

\section{Surgical considerations and technique}

The tailored minimal invasive technique used by the senior author has been previously described in detail [24].

The choice of the nostril used for the approach is determined by both the individual intranasal anatomy and by the existence of the lateral extension of the tumor (better view coming from the opposite nostril). Usually, a right nostril approach is generally preferred but a binostril approach can be used in difficult cases or in case of small nostrils.

Up to the opening of the sellar floor a short $0^{\circ}$ rigid endoscope hand held $(18 \mathrm{~cm}$ length, $4 \mathrm{~mm}$ diameter; KarlStorz, Tuttlingen, Germany) is used.

Using a blunt spatula the middle and superior turbinates are gently displaced to the lateral and the sphenoidal ostium is identified.

The mucosa from the sphenoid ostium to the base of the vomer is coagulated and divided using a blunt spatula. A medial sub mucosal dissection is performed identifying the junction between the vomer and the posterior bony septum and lateral pressure is applied on the septum in order to visualize the contralateral sphenoidal ostium. Once the sphenoidal rostum is clearly visible it is removed with a rongeur or forceps in between the two sphenoidal ostia

Mucosa of the sphenoid sinus is widely removed. The sphenoid sinus can have often multiple septations that have to be removed in order to clearly see the sphenoidal landmarks.

A long rigid $0^{\circ}$ endoscope $(30 \mathrm{~cm}$ length, $4 \mathrm{~mm})$ is used and is attached to the mechanic holder fixed on the operating table (Karl-Storz, Tuttlingen, Germany). The endoscope is placed in the upmost part of the sphenoid sinus, providing a panoramic field of view and ample workspace underneath.

The sellar floor can be opened either directly with a $1 \mathrm{~mm}$ rongeur (as is in the case of large macroadenomas where the sellar floor is reduced to a egg shell consistence), or by performing a downwards-oriented flap that can be replaced at the end of surgery. Sellar floor is usually opened anteriorly up to the tuberculum sellae, posteriorly to the

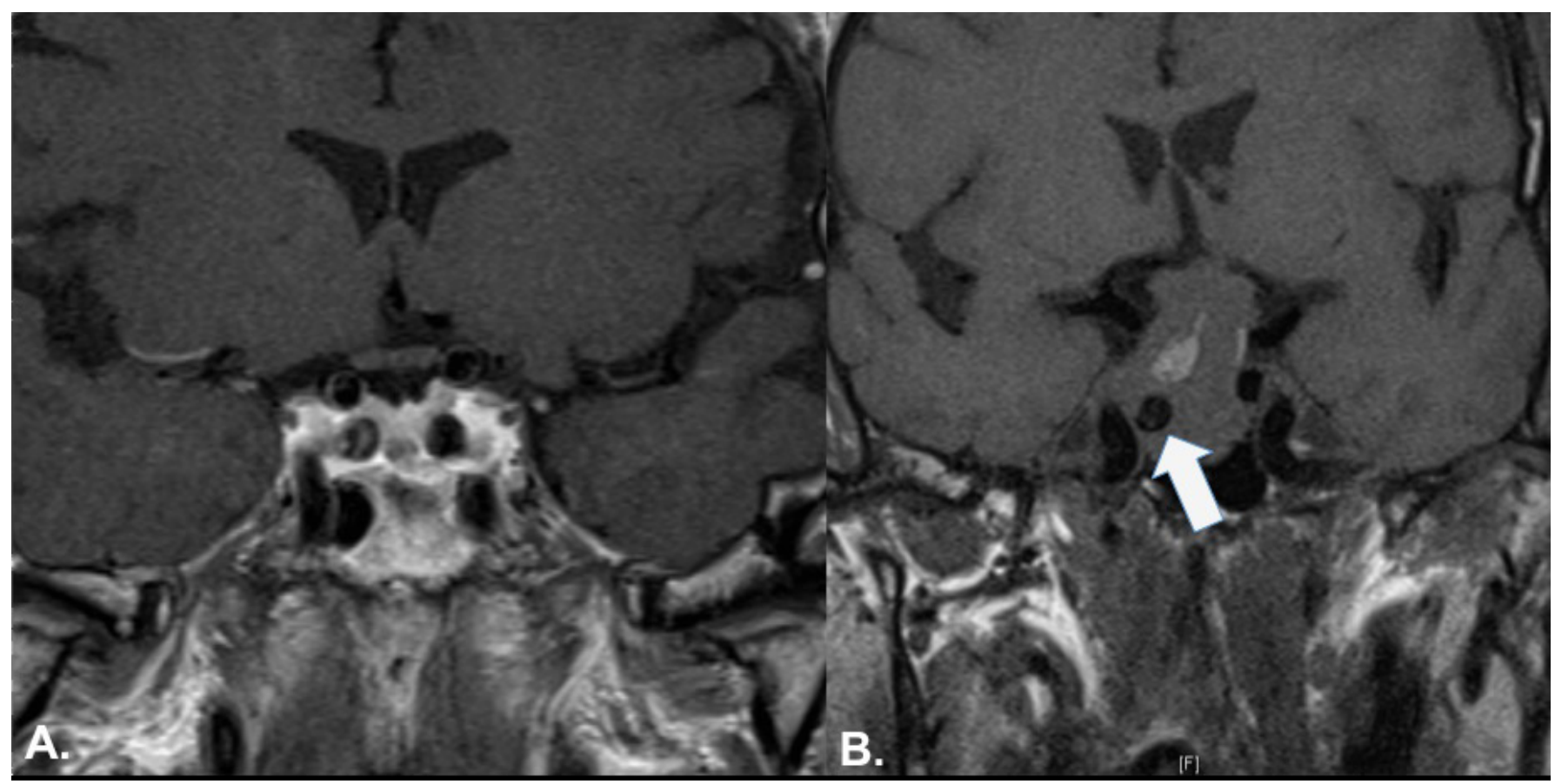

Fig. 3. IRM aspects of anomalous carotid encountered in patients with GH secreting adenomas: A. kissing carotids. B intrasellar procidence of right carotid artery. 
posterior intercavernous sinus and laterally to the cavernous sinus.

Dural opening is performed using a diamond tipped micro blade (Aesculap, Tuttlingen, Germany $y^{\bullet}$ ).

Removal of the adenoma is performed using angulated curettes and gentle suction in a piece meal fashion. Removal is started from the sellar floor and is then performed alongside the cavernous sinuses and lastly towards the sellar diaphragm. In the rare case of a firm pituitary adenoma extracapsular en block resection can be used.

After gross total removal, the sella is washed-out with saline solution and a $30^{\circ}$ short angled endoscope is used to inspect the intrasellar compartment for any residual tumor, especially toward the cavernous sinuses. A Valsalva maneuver is performed at this step in order to check for unrecognized CSF leak. The sellar phase is concluded with a thorough hemostasis. Reconstruction of the dural plane is performed using a dural substitute (Seamdura ${ }^{\circ}$, Gunze, Tokyo, Japan) placed extraduraly. Reinforcement is then performed using bioglue (Tissucol ${ }^{\circ}$, Baxter, Volketwil, Switzerland). If a bone flap was performed, it is then replaced and secured with bioglue. In cases without CSF leak the sphenoid sinus is left empty and no packing of the nasal cavities is performed.

When a CSF fistula is encountered, a multilayer closure technique is used with fat inside the sella, dural substitute extraduraly and fat and sometimes fascia lata to plug the sphenoid sinus reinforced by glue.

\section{Discussions}

\section{Steps taken in order to avoid and manage complications}

\section{Preoperative imaging}

Detailed information of the anatomy is the best way to prevent any kind of complications during a transshpenoidal approach, but is especially useful for the avoidance of vascular complications [25].

All patients have prior to surgery at least two MRI examinations. One a typical targeted examination of the pituitary region including coronal and sagittal small field of view T2, T1 and T1 post contrast images. Dimensions of the tumor, invasion of the cavernous sinuses with the Knosp grading system, position of remaining pituitary, ICA route and conformation of the sellar diaphragm are being constantly noted. The other MRI examination is a 120-slice axial plane postcontrast neuronavigation sequence.

Skull base CT is used when particular bony anatomical traits, such as a multiseptated or less pneumatisated sphenoid sinus are found or for redo surgery. Other imaging sequences are such as angio-MRI or angiography are reserved for the cases with a suspicion of an intrasellar vascular malformation.

Careful inspection of these investigations should provide accurate information on the abnormalities encoun- tered in the nasal fossa (septal deviation, morphology of the turbinates), pneumatisation of the sphenoidal sinus, thickness of the sphenoid sinuses walls, septations and their positions relative to the carotid arteries, sellar morphology, positioning of the carotids, tumor size and tumoral invasion of the cavernous sinus, supradiaphragmatic space or sphenoid sinus. A particular attention should be directed to the intercarotid space.

\section{Anesthesia}

In order to reduce the bleeding it is of paramount importance that the systolic blood pressure be adequately controlled in the range of $100 \mathrm{~mm} \mathrm{Hg}$ by the anesthesiologist. This has to be coupled with deep anesthesia during the nasal phase, as this constitutes a particular painful part of the procedure[24].

\section{Neuronavigation and Doppler}

The use of neuronavigation systems has found wide acceptance for the transsphenoidal surgery. Image-fusion based navigation permits optimal visualization of bone, soft tissue and vascular structures during endoscopic pituitary surgery [3]. This improves the surgeon's orientation during the approach, helps identify sphenoidal septations and provides information on the location of the carotids and optic nerve.

Neuronavigation can be assisted with the use of a micro Doppler probe to insonate the carotids, providing an additional safety layer to the approach. Proper anatomical and surgical judgment should be always used as lack of Doppler signal does not directly imply the absence of the carotid [26].

\section{Tips for avoidance and management of intraoperative vascular complications}

Judicious use of the surgical technique is essential to the avoidance of intraoperative complications. The surgeon should not attempt to perform surgical maneuvers in poor visual conditions or rush the surgical procedure in any condition.

\section{Hemorrhage from the mucosa and turbinates}

The nasal mucosa is very well vascularized and bleeds easily. Care should be taken to avoid a prolonged nasal phase or aggressive manipulation of the mucosa as this can lead to sustained early bleeding and increase the overall difficulty of the procedure.

If there is excessive hemorrhage from the mucosa, light packing with cottonoids soaked with diluted xylocaine and adrenaline can be performed and kept for several minutes with very good results.

Sustained hemorrhage from the mucosa can be also a sign of superficial anesthesia, asking the anesthesiologist to control the blood pressure and deepen the anesthesia usually significantly improves the working conditions.

When performing the lateralization the middle turbinate pressure needs to be gently placed along all the entire 
length of the middle turbinate in order to avoid their fracture, which leads to hemorrhage.

\section{Hemorrhage from branches of sphenopalatine artery}

Nasoseptal branches of the sphenopalatine artery are coursing on the superior margin of the choana. Instead of being just displaced inferiorly with the mucosa the nasoseptal branches should be coagulated in order to avoid hemorrhage from it. An extensive bipolar coagulation should be performed $10-15 \mathrm{~mm}$ vertically from the choana and medially to the ipsilateral sphenoid ostium, before proceeding with the submucosal dissection. This will ensure proper control of the sphenopalatine branches and greatly reduces the risk of postoperative epistaxis (Figure 1).

When injured, bipolar coagulation of the artery should be precise and persistent in order to reduce the risk of postoperative epistaxis. Coagulation must be done on a sufficient length of the artery before the spheno-palatine bony canal, otherwise the artery can retract inside and uncontrollable postoperative epistaxis can occur. It is often overlooked the fact that during the sphenoidothomy the contralateral nasoseptal branch can also be injured, this should also be checked and coagulated if needed.

The incidence of postoperative epistaxis (minor and major) following pituitary surgery varies between $0-7 \%$ [27] and can occur mainly during the first 2 weeks, rarely after.

In case of minor postoperative epistaxis, digital compression or nasal packing has to be performed on the side of bleeding.

Major delayed epistaxis is a rare but life threatening event requiring immediate action. Patency of respiratory tract needs to be assessed. Rarely, but if required, orotraheal intubation should be performed. Coagulopathies if present are to be treated aggressively. Blood pressure has to be kept in normal ranges in order to avoid ischemic complications.

The main cause of major epistaxis is vascular scar from an sphenopalatine artery falling down or a ruptured of a psuedoaneurysm of the spheno-palatine branches [28]. However, an ICA injury must always be rulled out by performing an angiography.

A bilateral nasal packing is mandatory and if is not enough to stop bleeding, our preference is to ask neuroradiologist for an embolization on the side of bleeding and sometimes bilaterally if needed. Embolization of the maxillary artery or the external carotid provides adequate long term control of the hemorrhage [29].

The alternative is a redo surgery to ligature or coagulate the arterial branch but embolization if available is faster and does not require a general anesthesia, which is poses risks during intubation when bleeding is severe.

After embolization, the packing is deflated at day 1 and removed at day 2 with a nasal inspection few days after.

\section{Injury to the internal carotid artery (ICA)}

Prevention: Proper localization of the neurovascular sphenoidal landmarks is the most important step in avoiding injury to the carotid artery.
Abnormal ICA route can be encountered in quite often in some patients (tortuous or kissing arteries in acromegaly patients, ICA procidence), it is of upmost importance that these variations should be searched during the preoperative planning.

Endoscopic beginners should be aware that the large field of view of the endoscope could push surgeon to have a large bone aperture increasing the risk of ICA damage. In that way, surgeon must keep intact the ICA C5 bone canal. In case of bone aperture around the ICA or cavernous sinus (a situation rarely mandatory when a extended lateral surgery is chosen), the bone must be split from the dura and out fractured using bone punches rather than removing with a piecemeal technique.

Dural opening should be performed in " $\mathrm{X}$ " or " $\mathrm{H}$ " fashion, starting superiorly and lateral and moving inferiorly and medial, keeping the cutting part of the blade oriented towards the midline. In this fashion the blade is always oriented away from the closest point of the ipsilateral carotid and accidents can be avoided. It is possible for the surgeon to perform a small medial dural opening that can be enlarged with curettes and other blunt dissecting instruments. A limited lateral incision can be performed in the case of lateralized microadenomas. The " $\mathrm{X}$ " or " $\mathrm{H}$ " incision also has the advantage of providing adequate control of the anterior intercavernous sinus.

During the intrasellar dissection, only blunt curettes or suction had to be used and blind manipulation inside the cavernous sinus is strictly forbidden.

Treatment. Injury of the carotid artery during an endoscopic transsphenoidal approach is a rare $(0.2-1 \%)$ but devastating complication [10], posing the patient in an acute vital risk. This has been shown to be clearly rare in experimented hands [30].

There is no universal protocol of how the management of this complication should be performed, but some important points that should be noted:

When ICA injury arrives during the sphenoidal step, the injury locates at the C5 ICA segment in most of cases. Packing is therefore the only way to control the bleeding and as the bony structures are intact, over plugging will not be dangerous or compressive. It might be useful to use 2 suctions with the help of the assistant as bleeding blinds quickly the surgical field. When carotid injury is occurs inside the sella, intraduraly, every attempt has to be made to avoid any compressive over packing.

There are also anecdotic reports on the use of harvested and crushed muscle on the site of injury or direct clipping with endoscopic vascular clamp [31]. These techniques are based on animal experimental models, and it is our opinion that surgeons should not loose time in the pursuit of such actions.

If adequate control is not achieved the patient has to be patient rushed to for emergency endovascular intervention.

If the intraoperative control of hemorrhage is successful, immediate angiography is mandatory and endovascular intervention is most likely required. 
Decision of ICA management is made both by the neurosurgeon and the radiologist. If there is a functional Polygon of Willis, occlusion is the ICA is more reliable. If not, stenting and coiling must be performed (Figure 4).

In some situations with less dramatic hemorrhage, any ICA injury can be found at the angiography. It might be important to stress out that injury an inferior pituitary branches can mimic an ICA issue.

In order to adequately asses the risk of a posttraumatic pseudo-aneurysm, controls should be performed at 1 week, 6 weeks, 3 months and 1 year [31].

Other arterial origin of bleeding: trigeminal artery, inferior hypophyseal artery or intrasellar aneurysm.

The coexistence of a pituitary adenoma and an intrasellar vascular malformation be it aneurysm or vascular vestigial remnant is extremely rare. The added vascular complex- ity makes surgery extremely risky. Preoperative lookup is the best way to avoid these insidious traps. Surgery should be delayed until effective endovascular procedures have resolved the vascular pathology.

Hemorrhage from the inferior hypophyseal artery can be sometimes encountered requiring adequate coagulation or packing. Postoperative care should be taken as obliteration of the inferior hypophyseal artery has been linked with apoplexy or diabetes insipidus [32,33].

\section{Tumor hemorrhage}

Hemorrhage from the tumor can be stopped in most situations time by simple cottonoid application and irrigation with warm saline solution. The use of intrasellar contact hemostatic agents is reserved for cases with profuse hemorrhage.

In the case of adenomas infiltrating the cavernous sinus, resection of the adenoma should not be performed if man-

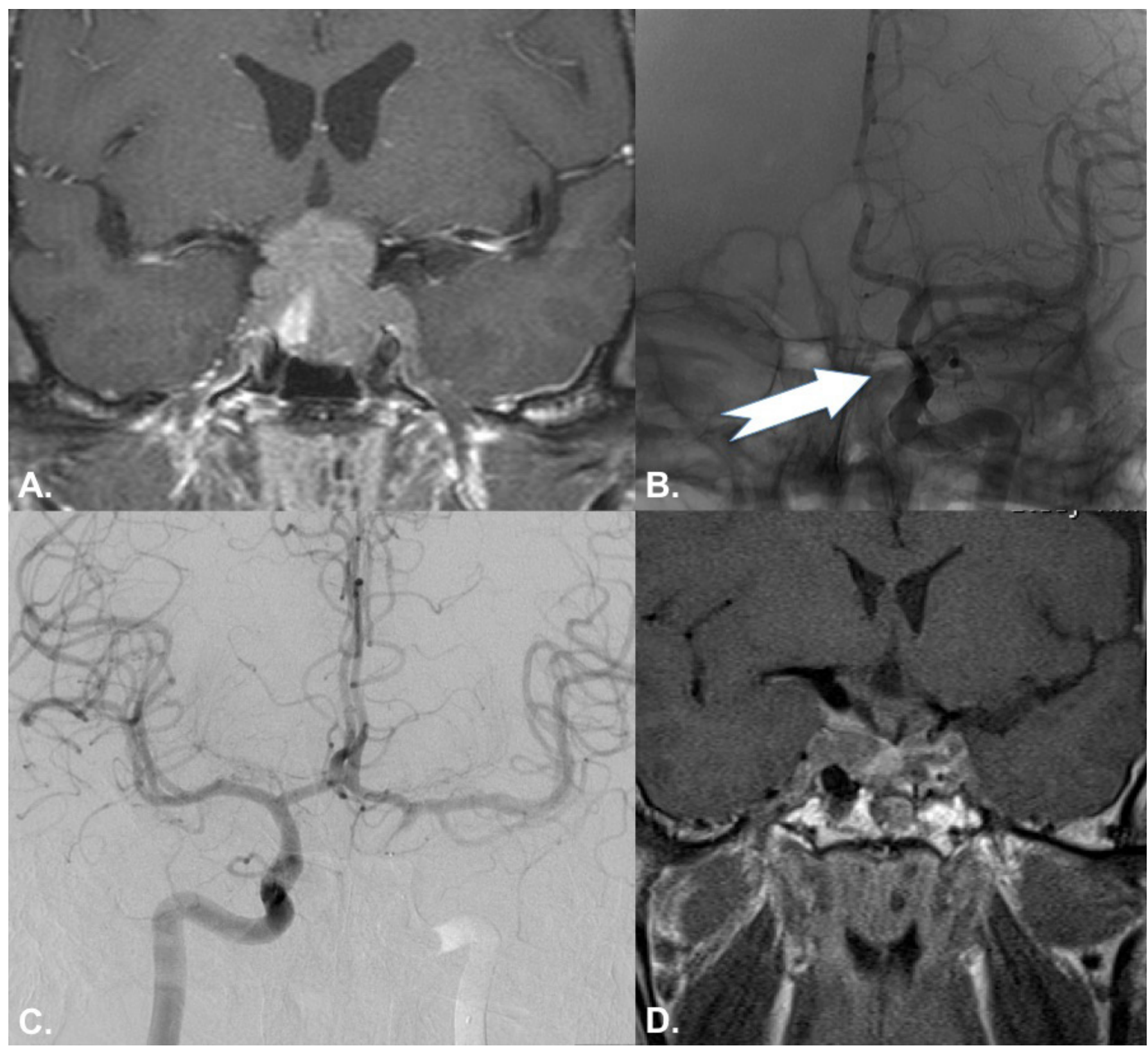

Fig. 4. Accidental injury of the left internal carotid injury in $54 \mathrm{yr}$ old male. Preoperative image. Partial occlusion with gauze packing of the C4 segment, left internal carotid, after intradural injury of the left intercavernous carotid artery. Left carotid balloon occlusion, with patent Circle of Willis. Control IRM after redo surgery performed 2 years after vascular injury. 
datory (which is currently rare) with the use of curettes, but instead a double hand two suctions technique should be used under visual control. The use of gentle double suction provides for a better tumor control without the risk of using blunt instruments that can perforate the thin cavernous wall.

\section{Venous bleeding}

In cases with a significant hemorrhage from a patent anterior intercavernous sinus hemostasis is obtained by coagulation or use of micro hemostatic clips.

Posterior intercavernous sinus is often opened up during sellar exploration for Cushing adenomas and can be easily managed by simple cottonoid compresstion.

Bleeding from the cavernous sinus that can be opened up during microadenoma surgery can be handled with the use of contact hemostatic agents or solvent hemostatic agents (FloSeal ${ }^{\bullet}$, Baxter, Volketwil, Switzerland).

\section{Conclusion}

Transnasal approaches for pituitary adenomas have become the standard of treatment in this pathology for the last 40 years. Since 2 decades, endoscopy brings a panoramic field of view, a wider surgical field with important vascular structures now reachable; this allows for control but also may bring higher risk.

As more centers start to implement this procedure it is vitally important to underline that a good knowledge of the skull base anatomy, good quality imaging sequences and use of a proper surgical technique can easily and greatly reduce the vascular risks of this surgery.

\section{Acknowledgement}

This paper was published under the frame of European Social Found, Human Resources Development Operational Programme 2007-2013, project no. POSDRU/159/1.5/S/136893

\section{References}

1. Jho HD, Carrau RL. Endoscopic endonasal transsphenoidal surgery: experience with 50 patients. J Neurosurg. 1997;87:44-51.

2. Gondim J a, Almeida JPC, Albuquerque LAF, et al. Endoscopic endonasal approach for pituitary adenoma: surgical complications in 301 patients. Pituitary. 2011;14:174-83.

3. Mert A, Micko A, Donat M, et al. An advanced navigation protocol for endoscopic transsphenoidal surgery. World Neurosurg 2014;82:S95105.

4. Yano S, Shinojima N, Kawano T, et al. Endoscopic endonasal skull base approach for parasellar lesions: Initial experiences, results, efficacy, and complications. Surg Neurol Int. 2014;5:51.

5. Cappabianca P, Cavallo LM, Solari D, et al. Endoscopic endonasal surgery for pituitary adenomas. World Neurosurg. 2014;82:S3-11.

6. Ammirati M, Wei L, Ciric I. Short-term outcome of endoscopic versus microscopic pituitary adenoma surgery: a systematic review and metaanalysis. J Neurol Neurosurg Psychiatry. 2013;84:843-9.

7. Warnke PC. Case series analysis, meta-analysis or no analysis in the evaluation of neurosurgical techniques: get better or get out. J Neurol
Neurosurg Psychiatry. 2013;84:828

8. Laws ER. Complications of transsphenoidal surgery: the shortcomings of meta-analysis. J Neurol Neurosurg Psychiatry. 2013;84:829.

9. Berhouma M, Messerer M, Jouanneau E. Chirurgie endoscopique de I'hypophyse et de la base du crâne. EMC - Neurol. 2013;10:1-20.

10. Berker M, Hazer DB, Yücel T, et al. Complications of endoscopic surgery of the pituitary adenomas: analysis of 570 patients and review of the literature. Pituitary. 2012;15:288-300.

11. Rhoton AL, Natori Y. The Orbit and Sellar Region: Microsurgical Anatomy \& Operative Approaches. Thieme, NY; 1997.

12. Unlu A, Meco C, Ugur HC, et al. Endoscopic anatomy of sphenoid sinus for pituitary surgery. Clin Anat. 2008;21:627-32.

13. Perondi GE, Isolan GR, de Aguiar PHP, Stefani MA, Falcetta EF. Endoscopic anatomy of sellar region. Pituitary. 2013;16:251-9.

14. Sareen D, Agarwal A, Kaul J, Sethi A. Study of sphenoid sinus anatomy in relation to endoscopic surgery. Int J Morphol. 2005.

15. Warnke J-P, Tschabitscher M, Thalwitzer J, Galzio R. Endoscopic anatomy for transnasal transsphenoidal pituitary surgery in the presence of a persistent trigeminal artery. Cent Eur Neurosurg. 2009;70:207-10.

16. Zada G, Agarwalla PK, Mukundan S, et al. The neurosurgical anatomy of the sphenoid sinus and sellar floor in endoscopic transsphenoidal surgery. J Neurosurg. 2011;114:1319-30.

17. Solari D, Chiaramonte C, Di Somma A, et al. Endoscopic anatomy of the skull base explored through the nose. World Neurosurg. 2014;82:S164-70.

18. Hamberger C, Hammer G, Marcusson G. Experiences in transantrosphenoidal hypophysectomy. Trans Pac Coast Otoophthalmol Soc Annu Meet. 1961;42:273-86.

19. Unal B, Bademci G, Bilgili YK, Batay F, Avci E. Risky anatomic variations of sphenoid sinus for surgery. Surg Radiol Anat. 2006;28:195-201.

20. Ebner FH, Kuerschner V, Dietz K, et al. Reduced intercarotid artery distance in acromegaly: pathophysiologic considerations and implications for transsphenoidal surgery. Surg Neurol. 2009;72:456-60; discussion 460.

21. Machado MC, Kodaira S, Musolino NRC. Persistence of intrasellar trigeminal artery and simultaneous pituitary adenoma: description of two cases and their importance for the differential diagnosis of sellar lesions. Arq Bras Endocrinol Metabol. 2014;58:661-5.

22. Klose S, Kopf D, Lehnert H. Giant intrasellar carotid aneurysm - an unusual cause of panhypopituitarism. Exp Clin Endocrinol Diabetes. 2005;113:551-3.

23. Sade B, Mohr G, Tampieri D, Rizzo A. Intrasellar aneurysm and a growth hormone-secreting pituitary macroadenoma. Case report. J Neurosurg. 2004;100:557-9.

24. Berhouma M, Messerer M, Jouanneau E. Occam's razor in minimally invasive pituitary surgery: tailoring the endoscopic endonasal uninostril trans-sphenoidal approach to sella turcica. Acta Neurochir (Wien). 2012;154:2257-65.

25. Laws ER. Vascular complications of transsphenoidal surgery. Pituitary. 1999;2:163-70.

26. Dusick JR, Esposito F, Malkasian D, Kelly DF. Avoidance of carotid artery injuries in transsphenoidal surgery with the Doppler probe and microhook blades. Neurosurgery. 2007;60:322-8; discussion 328-9.

27. Griffiths CF, Cutler AR, Duong HT, et al. Avoidance of postoperative epistaxis and anosmia in endonasal endoscopic skull base surgery: A technical note. Acta Neurochir (Wien). 2014;156:1393-401.

28. Raymond J, Hardy J, Czepko R, Roy D. Arterial injuries in transsphenoidal surgery for pituitary adenoma; the role of angiography and endovascular treatment. AJNR Am J Neuroradiol. 1997;18:655-65.

29. McClurg SW, Carrau R. Endoscopic management of posterior epistaxis: a review. Acta Otorhinolaryngol Ital. 2014;34:1-8.

30. Ciric I, Ragin A, Baumgartner C, Pierce D. Complications of transsphenoidal surgery: results of a national survey, review of the literature, and personal experience. Neurosurgery. 1997;40:225-36; discussion236-7.

31. Padhye V, Valentine R, Wormald P-J. Management of Carotid Artery Injury in Endonasal Surgery. Int Arch Otorhinolaryngol. 2014;18:S173-8.

32. Nawar RN, AbdelMannan D, Selman WR, Arafah BM. Pituitary tumor apoplexy: a review. J Intensive Care Med. 23:75-90.

33. Phatouros CC, Higashida RT, Malek AM, et al. Embolization of the meningohypophyseal trunk as a cause of diabetes insipidus. Am $J$ Neuroradiol. 1999;20:1115-8. 